\title{
Ligament Reconstruction in Congenital Absence of the Anterior Cruciate Ligament: A Case Report
}

\author{
Jae Jeong Lee, MD, Won Taek Oh, MD, Keun Young Shin, MD, Min Seok Ko, MD and \\ Chong-Hyuk Choi, MD, PhD \\ Department of Orthopedic Surgery, Gangnam Severance Hospital, Yonsei University College of Medicine, Seoul, Korea
}

Congenital absence of the cruciate ligament is an extremely rare condition that was first reported in Giorgi's radiographic study in 1956. The authors report on a case of anterior cruciate ligament reconstruction performed on a 21-year-old female patient with congenital anterior cruciate ligament absence. We also discuss radiographic evidence that could provide clues to the congenital absence and possible difficulties that may be encountered during surgery with a review of the relevant literature.

Key words: Anterior cruciate ligament, Congenital absence, Anterior cruciate ligament reconstruction.

Congenital absence of the cruciate ligament is an extremely rare condition with a prevalence of 0.017 per 1,000 live births, which was first reported in Giorgi's ${ }^{1)}$ radiographic study in 1956. Congenital absence of the anterior cruciate ligament (ACL) may present as an independent defect ${ }^{2,3)}$ or as part of a complex syndrome $e^{4,5)}$.

The first domestic report of a patient with ACL deficiency was made in 1997 by Park et al. ${ }^{6}$. In that study, the authors just performed arthroscopic examination and not reconstruction. This study is on a case of ligament reconstruction performed on a 21 -year-old female congenital ACL absence patient who complained of subjective instability and presented with objective instability of the knee joint. We discuss radiographic evidence

Received July 18, 2011; Revised (1st) August 18, 2011;

(2nd) September 15, 2011; Accepted September 20, 2011.

Correspondence to: Chong-Hyuk Choi, MD, PhD.

Department of Orthopedic Surgery, Gangnam Severance Hospital,

Yonsei University College of Medicine,

146-92 Dogok-dong, Gangnam-gu, Seoul 135-720, Korea.

Tel: +82-2-2019-3415, Fax: +82-2-573-5393

Email: choi8422@yuhs.ac

This is an Open Access article distributed under the terms of the Creative Commons Attribution Non-Commercial License (http://creativecommons.org/licenses/by-nc/3.0/) which permits unrestricted non-commercial use, distribution, and reproduction in any medium, provided the original work is properly cited.

Copyright ( ) 2011. KOREAN KNEE SOCIETY

www.jksrr.org that could provide clues to this congenital absence and possible difficulties that may be encountered during surgery with a review of the relevant literature.

\section{Case Report}

A 21-year-old female patient presented with instability of the left knee joint. She had no history of trauma but a continued feeling of instability during ambulation and experienced a feeling of giving way about two to three times a month. A physical examination showed no tenderness or swelling, and the range of motion was normal. The patient displayed some positive signs, grade 3 on Lachman's test and the anterior drawer test and grade 2 on the pivot shift test. The side to side difference in the anterior drawer test using the KT-2000 arthrometer was $15.3 \mathrm{~mm}$.

The distal medial condyle of the left femur appeared hypoplastic on conventional radiographs. On scanogram, the left femur (37.8 $\mathrm{cm}$ ) was shorter than the right femur $(39.4 \mathrm{~cm})$, while the left and right tibiae were the same length. Anterior and posterior simple radiographs showed hypoplasia of the left lateral tibial spine and a lateral view showed a decreased posterior tibial slope compared to that of the right side. On the Knee Lachman stress view, the side to side difference between each anterior drawer was determined to be $16.7 \mathrm{~mm}$ (Fig. 1).

The ACL was not visible on magnetic resonance imaging (MRI) scans and the posterior cruciate ligament (PCL) also seemed hypoplastic. The medial femoral condyle appeared comparatively smaller, the intercondylar notch was decreased in height and 

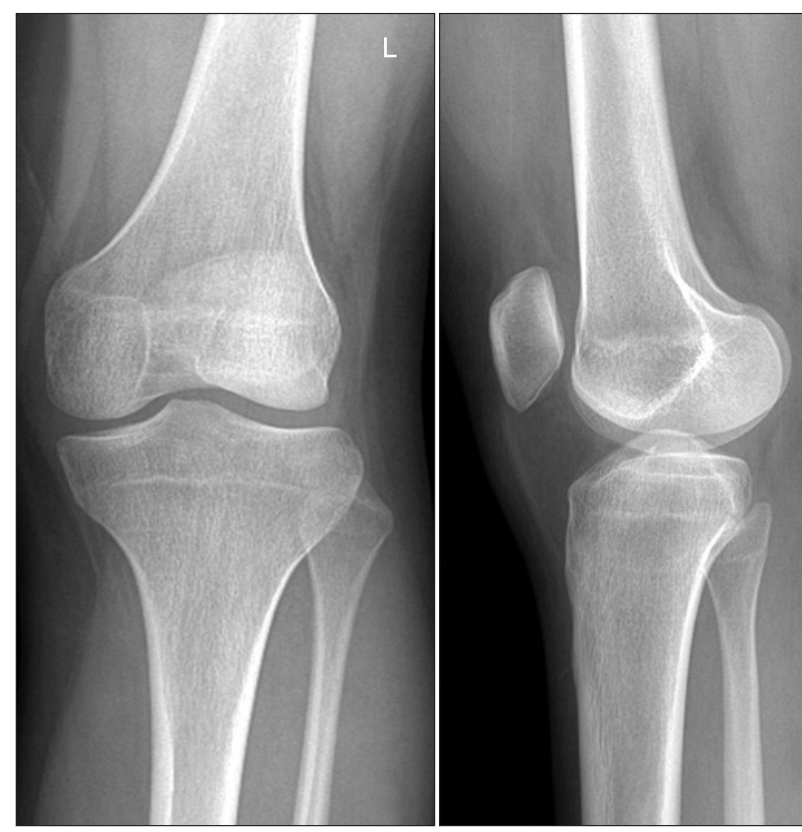

L
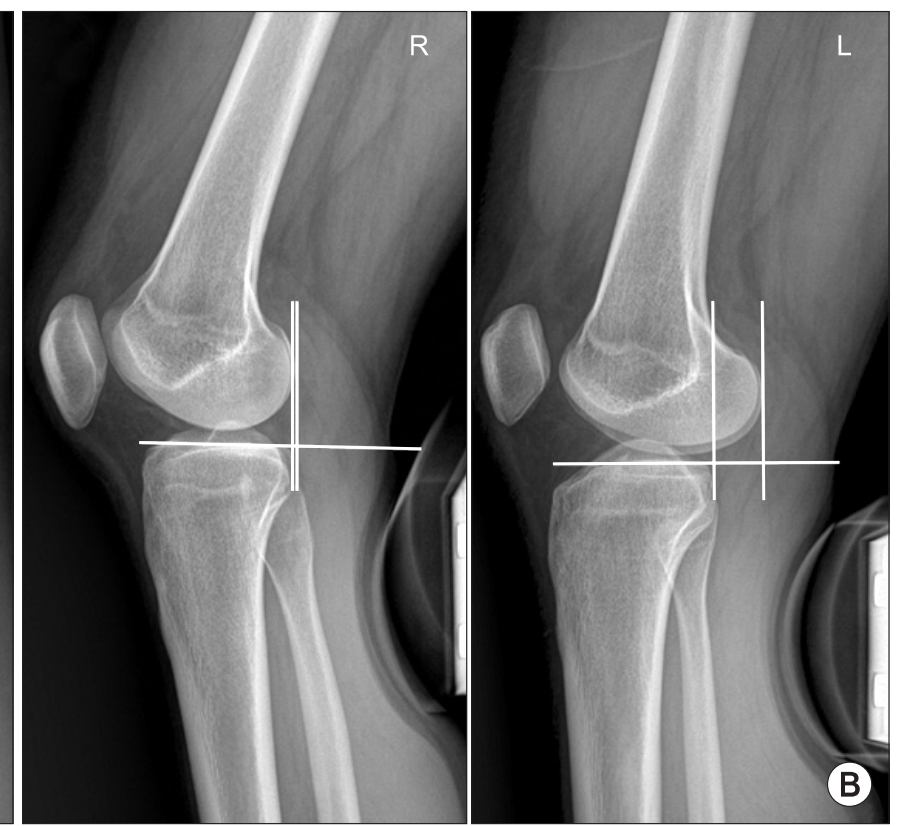

Fig. 1. (A) Standing anteroposterior and lateral radiographs show lateral tibial spine hypoplasia and decreased posterior slope of the left knee. (B) Knee Lachman stress view shows $16.7 \mathrm{~mm}$ more anterior translation of the left knee compared to the right knee.
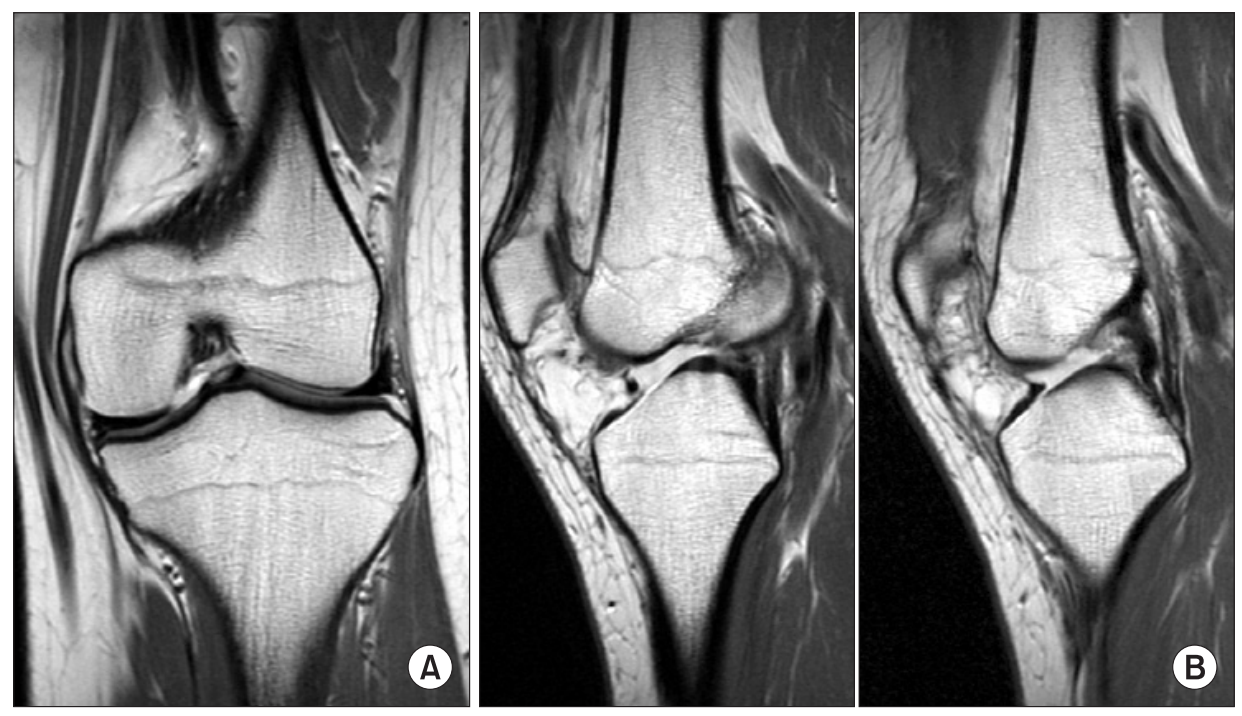

Fig. 2. (A) Hypoplasia of the medial femoral condyle and narrow intercondylar notch are seen in the coronal view of the left knee magnetic resonance imaging scan. (B) Absence of the anterior cruciate ligament and hypoplasia of the posterior cruciate ligament are seen in the sagittal view of the left knee magnetic resonance imaging scans.

width, and the cartilaginous covering of the lateral intercondylar notch appeared broader compared to normal specimens (Fig. 2).

Reconstructive surgery of the cruciate ligament was planned and arthroscopy was performed. The arthroscopy showed that the ACL was absent, the PCL was maintained, and the lateral femoral intercondylar notch was broadly covered with cartilage as seen on the preoperative MRI. An incomplete longitudinal tear, about $5 \mathrm{~mm}$ in size, was observed at the posterior horn of the medial meniscus. It was left in situ because it did not appear to be pathological. Other defects or abnormal signs were not observed.
Notchplasty was extensively performed to widen the abnormally narrow femoral intercondylar notch, after which transtibial single-bundle ACL reconstruction was performed using an allograft of the anterior tibialis tendon (Fig. 3). A tibial footprint of $10 \mathrm{~mm}$ in diameter was created on the tibial footprint of the ACL. Then, a femoral socket $10 \mathrm{~mm}$ in diameter was created at the 1 oclock position using reamers and dilators with the knee at $90^{\circ}$ of flexion. The transplanted tendon was fixed to the proximal tibia using an interference screw and to the distal femur using two Rigidfix Cross Pin devices (DePuy Mitek, Raynham, MA, 

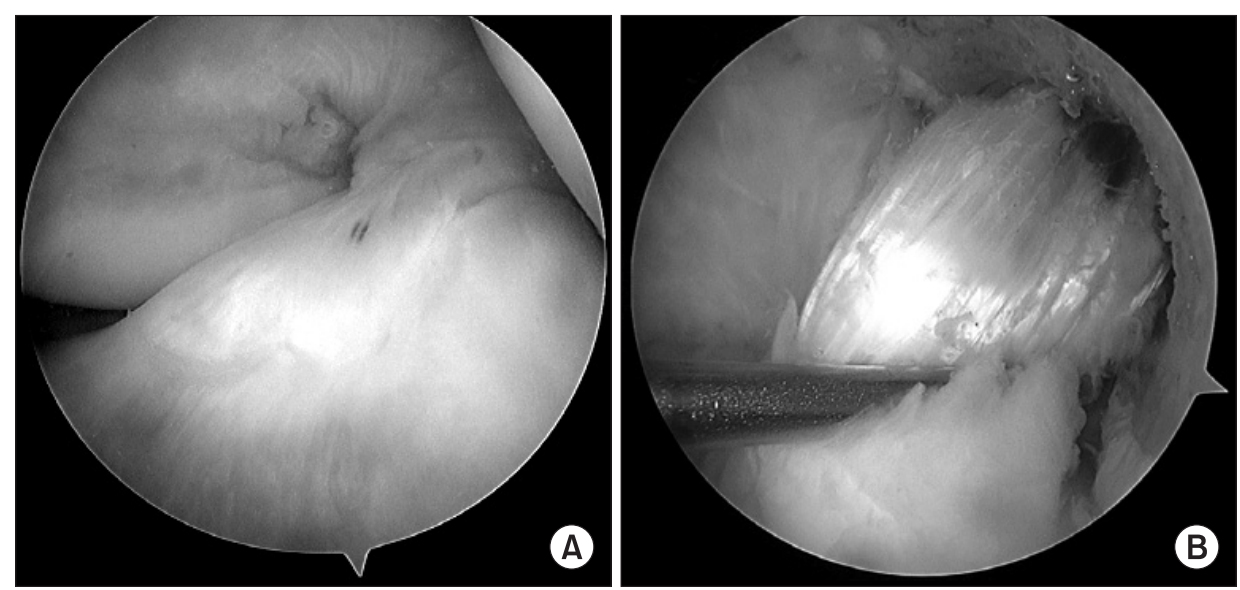

Fig. 3. (A) An arthroscopic image of the knee shows absence of the anterior cruciate ligament (ACL) within a narrow intercondylar notch. (B) An extensive notchplasty was performed due to the narrow intercondylar notch and an ACL reconstruction using an allo- anterior tibialis tendon was done.

USA). The reconstruction was completed after normal range of motion and stability of the knee joint were confirmed.

The patient was followed up for 6 months after the knee reconstruction. Examination of the knee range of motion revealed no limitation. The side to side difference in the anterior drawer test using the KT-2000 arthrometer was $3.8 \mathrm{~mm}$ and the pivot shift test was negative.

\section{Discussion}

Congenital absence of the cruciate ligament is an extremely rarely reported condition. Patients often adjust to their congenital knee condition and mostly do not complain of subjective instability or pathologic instability despite showing positive results on objective instability tests (e.g., Lachman test, anterior/ posterior drawer test, pivot shift test ${ }^{7)}$. It has been known that patients most often complain of pain due to arthritis of the medial femorotibial joint or the patellofemoral joint, which progresses more slowly than the arthritis caused by traumatic ACL rupture ${ }^{3)}$.

When the contralateral knee is normal and there is a history of trauma, it is difficult to clinically distinguish congenital absence of the ACL from traumatic ACL rupture. There are several radiographic signs that could be clues to a diagnosis of congenital absence of the ACL and the most widely known and easily observed of these is hypoplasia of the tibial spine. ${ }^{3,8)}$ The second important clue is the shortening of the height and width of the intercondylar notch, which can be confirmed on a tunnel view radiographs or $\mathrm{MRI}^{8,9)}$. Manner et al. ${ }^{9)}$ classified congenital absence of the cruciate ligament into three types and delineated radiographic features of each type: type I is characterized by hypoplasia or aplasia of the ACL with a normal PCL; type II, by aplasia of the ACL in combination with hypoplasia of the PCL; and type III, by aplasia of both cruciate ligaments. Our case falls into type II and corresponds with their radiographic description, which is hypoplastic lateral tibial spine and narrow intercondylar notch.

Narrowing of the intercondylar notch may mean a limited field of view and space to work with during reconstruction, which should be taken into account during pre-operative planning. In particular, when the problem is combined with the absence of the PCL, the space becomes even smaller and the likelihood of operative failure increases ${ }^{8)}$. Additionally, the lateral femoral intercondylar notch is widely covered with cartilage ${ }^{9)}$, which was confirmed by MRI preoperatively and by arthroscopy during the operation in our case. There have been reports in the literature of increases in the posterior slope angle, hypoplasia of the lateral femoral condyle, and abnormally rounded posterior curvature of the femoral condyle. ${ }^{3)}$ The latter was not observed in our case but hypoplasia of the medial condyle was.

Some malformations associated with congenital absence of the ACL are abnormalities of the menisci, thrombocytopeniaabsent radius syndrome, congenital knee dislocation, and various congenital longitudinal deficiencies of the lower limb including proximal femoral focal deficiency ${ }^{4,5}$. In this case, none of these malformations were observed. The patient's left leg was shortened compared to the right leg, but not to an extent that would require correction.

The necessity of ligament reconstruction for congenital absence of the ACL has been under dispute for several reasons, the first being that patients often adjust to their congenital absence and do not feel discomfort in their activities of daily living and the second being the technical difficulty of the procedure and its high postoperative failure rate ${ }^{10)}$. Finally, it is also controversial to what extent such a procedure can actually restore normal kinematics of a joint that is congenitally malformed in the first place. We 
are of the opinion that in cases where patients subjectively feel instability of the knee joint and show objective instability on tests such as the pivot shift test, ligament reconstruction should be performed in order to prevent further progression to arthritis.

\section{References}

1. Giorgi B. Morphologic variations of the intercondylar eminence of the knee. Clin Orthop. 1956;8:209-17.

2. Noble J. Congenital absence of the anterior cruciate ligament associated with a ring meniscus. J Bone Joint Surg Am. 1975; 57:1165-6.

3. Frikha R, Dahmene J, Ben Hamida R, Chaieb Z, Janhaoui N, Laziz Ben Ayeche M. Congenital absence of the anterior cruciate ligament: eight cases in the same family. Rev Chir Orthop Reparatrice Appar Mot. 2005;91:642-8.

4. Johansson E, Aparisi T. Missing cruciate ligament in congenital short femur. J Bone Joint Surg Am. 1983;65:110915.
5. Gabos PG, El Rassi G, Pahys J. Knee reconstruction in syndromes with congenital absence of the anterior cruciate ligament. J Pediatr Orthop. 2005;25:210-4.

6. Park SR, Kim HS, Kang JS, Lee WH, Lee JH, Lee TJ. Bilateral congenital deficiency of the anterior cruciate ligament. J Korean Arthrosc Soc. 1997;1:108-11.

7. Markolf KL, Kochan A, Amstutz HC. Measurement of knee stiffness and laxity in patients with documented absence of the anterior cruciate ligament. J Bone Joint Surg Am. 1984;66:242-52.

8. Balke M, Mueller-Huebenthal J, Shafizadeh S, Liem D, Hoeher J. Unilateral aplasia of both cruciate ligaments. J Orthop Surg Res. 2010;5:11.

9. Manner HM, Radler C, Ganger R, Grill F. Dysplasia of the cruciate ligaments: radiographic assessment and classification. J Bone Joint Surg Am. 2006;88:130-7.

10. Carlioz H. Description and natural history of severe aplasia of the lower extremities. Chir Pediatr. 1978;19:306-21. 